Research Articles: Behavioral/Cognitive

\title{
Chronic behavioral manipulation via orally delivered chemogenetic actuator in macaques
}

https://doi.org/10.1523/JNEUROSCI.1657-21.2021

Cite as: J. Neurosci 2022; 10.1523/JNEUROSCI.1657-21.2021

Received: 14 August 2021

Revised: 21 December 2021

Accepted: 23 December 2021

This Early Release article has been peer-reviewed and accepted, but has not been through the composition and copyediting processes. The final version may differ slightly in style or formatting and will contain links to any extended data.

Alerts: Sign up at www.jneurosci.org/alerts to receive customized email alerts when the fully formatted version of this article is published.

Copyright (c) 2022 Oyama et al.

This is an open-access article distributed under the terms of the Creative Commons Attribution 4.0 International license, which permits unrestricted use, distribution and reproduction in any medium provided that the original work is properly attributed. 
Oyama et al.

1 Chronic behavioral manipulation via orally delivered

3

4 Running title: Chronic chemogenetic control of monkey behavior

5

6

Kei Oyama ${ }^{1}$, Yukiko Hori ${ }^{1}$, Yuji Nagai ${ }^{1}$, Naohisa Miyakawa ${ }^{1}$, Koki Mimura ${ }^{1}$,

Toshiyuki Hirabayashi ${ }^{1}$, Ken-ichi Inoue ${ }^{2,3}$, Masahiko Takada ${ }^{2}$, Makoto Higuchi ${ }^{1}$,

Takafumi Minamimoto ${ }^{1 *}$ and Technology

${ }^{2}$ Systems Neuroscience Section, Primate Research Institute, Kyoto University, Inuyama, Aichi 484-8506, Japan

* Author to whom all correspondence should be addressed:

Takafumi Minamimoto, Ph. D. and Technology

4-9-1 Anagawa, Inage-ku, Chiba 263-8555 Japan

Phone: +81-43-206-4699

Fax: +81-43-253-0396

E-mail: minamimoto.takafumi@qst.go.jp (T.M.)

Number of pages: 48

Number of figures, tables, multimedia, and 3D models (separately): 6 figures, 1

table

\section{chemogenetic actuator in macaques \\ Chronic behavioral manipulation via orally delivered}

${ }^{1}$ Department of Functional Brain Imaging, National Institutes for Quantum Science

${ }^{3}$ PRESTO, Japan Science and Technology Agency, Kawaguchi, Saitama, Japan

Department of Functional Brain Imaging, National Institutes for Quantum Science 
28 Number of words for abstract, introduction, and discussion (separately): abstract,

29188 words, introduction, 550 words, discussion, 1499 words

30

31 Conflict of interest statement: Y.N., N.M., M.H. and T.M. are named as inventors on 32 a patent application (PCT/JP2019/024834; status: patent pending) claiming subject 33 matter related to the results described in this paper. The remaining authors declare 34 no competing interests.

35

Acknowledgments: This study was supported by MEXT/JSPS KAKENHI Grant 37 Numbers JP18K15353 and JP21K07268 (to KO), JP17H02219 (to TH), 38 JP19K07811 and JP20H04596 (to NM), JP19H05467 (to MT), JP19K08138 (to $39 \mathrm{YN}$ ), and JP18H04037 (to TM), by AMED Grant Numbers JP21dm0307007 (to $40 \mathrm{TH}$ ), JP20dm0307021(to KI), JP21dm0207077 (to MT), JP21dm0107146 (to TM), 41 by JST PRESTO Grant Number JPMJPR1683 (to KI), by QST President's 42 Strategic Grant (Creative Research)(to NM), by the cooperative research program 43 (2021-A-14) at PRI, Kyoto Univ., and by National Bio-Resource Project "Japanese 44 Monkeys" of MEXT, Japan. We thank J. Kamei, R. Yamaguchi, Y. Matsuda, Y. 45 Sugii, T. Okauchi, T Kokufuta, M. Fujiwara and M. Nakano for their technical 46 assistance. 


\section{Abstract}

The chemogenetic technology referred to as designer receptors exclusively activated by designer drugs (DREADDs) offers reversible means to control neuronal activity for investigating its functional correlation with behavioral action. Deschloroclozapine (DCZ), a recently-developed highly potent and selective DREADDs actuator, displays a capacity to expand the utility of DREADDs for chronic manipulation without side-effects in nonhuman primates, which has not yet been validated. Here we investigated the pharmacokinetics and behavioral effects of orally administered $\mathrm{DCZ}$ in female and male macaque monkeys. Pharmacokinetic analysis and positron emission tomography (PET) occupancy examination demonstrated that oral administration of $D C Z$ yielded slower and prolonged kinetics, and that its bioavailability was $10-20 \%$ of that in the case of systemic injection. Oral DCZ (300-1000 $\mu \mathrm{g} / \mathrm{kg})$ induced significant working memory impairments for at least $4 \mathrm{~h}$ in monkeys with hM4Di expressed in the dorsolateral prefrontal cortex (Brodmann's area 46). Repeated daily oral doses of DCZ consistently caused similar impairments over two weeks without discernible desensitization. Our results indicate that orally delivered DCZ affords a less invasive strategy for chronic but reversible chemogenetic manipulation of neuronal activity in nonhuman primates, and this has potential for clinical application. 
68

69

70

71

72

73

74

75

76

77

78

79

\section{Significance Statement}

The use of DREADDs for chronic manipulation of neuronal activity for days to weeks may be feasible for investigating brain functions and behavior on a long time-scale, and thereby for developing therapeutics for brain disorders, such as epilepsy. Here we performed pharmacokinetics and in vivo occupancy study of orally administered DCZ to determine a dose range suitable for DREADDs studies.

4 In monkeys expressing hM4Di in the prefrontal cortex, single and repeated daily doses significantly induced working-memory impairments for hours and over two 6 weeks, respectively, without discernible desensitization. These results indicate that 7 orally delivered DCZ produces long-term stable chemogenetic effects, and holds 8 great promise for the translational use of DREADDs technology. 


\section{Introduction}

The chemogenetic tool referred to as DREADDs (designer receptors exclusively activated by designer drugs) has been widely used for controlling neuronal activity and animal behavior in many species, including rodents and nonhuman primates (Roth, 2016). By combining mutated muscarinic receptors (excitatory hM3Dq and inhibitory hM4Di) with specific actuators such as CNO (clozapine-N-oxide), these chemogenetic techniques offer a means to remotely manipulate the activity of a specific neuronal population for minutes to hours (Urban and Roth, 2015). In addition to the acute control, repeated administration of the actuators enables chronic chemogenetic manipulation of neuronal activity for days to weeks (Grace et al., 2016; Nation et al., 2016; Wakaizumi et al., 2016; Andreoli et al., 2017; Yu and Münzberg, 2018; Desloovere et al., 2019; Paretkar and Dimitrov, 2019; Goossens et al., 2021), and thus has potential therapeutic applications. For example, chronic and reversible inhibition of neuronal activity can be an effective means for seizure control and epilepsy treatment (Lieb et al., 2019). For this purpose, oral delivery of agonists seems to be suitable, as it typically produces relatively long-lasting effects compared to systemic (i.e., intramuscular and intravenous) injections (Karbwang et al., 1997). Besides, oral administration is non-invasive and relieves the subject from stress during chronic treatment. It is also beneficial for neuroscience research aimed at long-term control of neuronal activity in freely-moving animals without restraint or stress. However, recent studies have reported that CNO has low permeability into the brain, and that its metabolite, clozapine, activates not only 
102

103

104

105

106

107

108

109

110

111

112

113

114

115

116

117

118

119

120

121

122

123

DREADDs but also endogenous receptors and transporters, leading to potential off-target effects (Gomez et al., 2017; Tran et al., 2020). To circumvent this issue, alternative selective DREADDs agonists have been explored and validated (Bonaventura et al., 2019; Weston et al., 2019; Nagai et al., 2020).

Deschloroclozapine (DCZ) is one of the 3rd-generation agonists for muscarinic DREADDs that represents high permeability into the brain and is highly potent and selective to DREADDs, as compared to prior agonists such as CNO and C21 (Nagai et al., 2020). Importantly, low doses of DCZ exert chemogenetic effects with rapid onset that last several hours in both rodents and nonhuman primates (Nagai et al., 2020). This indicates that DCZ is a promising chemogenetic actuator that can be used for a wide variety of objectives (e.g., Hirabayashi et al., 2021; Hori et al., 2021; Oyama et al., 2021). In particular, its superior efficacy and brain permeability permit its use for oral administration. In fact, it has been demonstrated that orally delivered DCZ effectively induces behavioral changes in mice and marmosets (Krause et al., 2021; Mimura et al., 2021). However, the longterm chemogenetic action of DCZ remains unclear, specifically in terms of the duration of a single-dosage effect and the impact of repetitive doses over several days to weeks. These will provide knowledge sufficient for designing chronic chemogenetic experiments and, in addition, for developing potential clinical therapeutic applications.

Here we investigated the pharmacokinetics and in vivo occupancy of orally administered DCZ to determine a dose range suitable for DREADDs studies in 
124 monkeys. We then demonstrated that oral DCZ administration induced a

125 chemogenetic behavioral effect lasting at least more than $4 \mathrm{~h}$ in macaque monkeys

126 with hM4Di expressed in the prefrontal cortex. We also found that repetitive daily

127 administrations of DCZ yielded consistent behavioral effects over weeks without

128 apparent signs of desensitization.

129

130 Materials and Methods

131 Subjects

132 A total of 12 macaque monkeys [10 Japanese (Macaca fuscata) and 2 Rhesus

133 monkeys (Macaca mulatta); 10 males, 2 females; 2.8-8.0 kg; age 4-10 years at the

134 beginning of experiments] were used (Table 1). All experimental procedures

135 involving animals were carried out in accordance with the Guide for the Care and

136 Use of Nonhuman primates in Neuroscience Research (The Japan Neuroscience

137 Society; https://www.jnss.org/en/animal_primates) and were approved by the

138 Animal Ethics Committee of the National Institutes for Quantum Science and

139 Technology. The monkeys were kept in individual primate cages in an air-

140 conditioned room (pair-housing environments are under preparation; see the

141 preface of the above guideline). A standard diet, supplementary fruits/vegetables

142 and a tablet of vitamin C (200 mg) were provided daily.

143

144 Viral vector production 
145

146

147

148

149

150

151

152

153

154

155

156

157

158

159

160

161

162

163

164 (cefmetazole, 25-50 mg/kg/day; ketoprofen, $1-2 \mathrm{mg} / \mathrm{kg} /$ day; 7 days) were

165 administered.

Adeno-associated virus (AAV) vector expressing hM4Di (AAV1-hSyn-hM4Di-IRES-

AcGFP, $4.7 \times 10^{13}$ particles $/ \mathrm{mL}$ ) was produced by helper-free triple transfection

procedure, which was purified by affinity chromatography (GE Healthcare,

Chicago, USA). Viral titer was determined by quantitative PCR using Taq-Man

technology (Life Technologies, Waltham, USA).

\section{Surgical procedures and viral vector injections}

Surgeries were performed under aseptic conditions in a fully equipped operating suite. We monitored body temperature, heart rate, $\mathrm{SpO}_{2}$ and tidal $\mathrm{CO}_{2}$ throughout

all surgical procedures. Monkeys were immobilized by intramuscular (i.m.) injection of ketamine $(5-10 \mathrm{mg} / \mathrm{kg})$ and xylazine $(0.2-0.5 \mathrm{mg} / \mathrm{kg})$ and intubated with an endotracheal tube. Anesthesia was maintained with isoflurane (1-3\%, to effect).

Before surgery, magnetic resonance (MR) imaging $(7$ tesla $400 \mathrm{~mm} / \mathrm{SS}$ system, NIRS/KOBELCO/Brucker) and X-ray computed tomography (CT) scans (Accuitomo170, J. MORITA CO., Kyoto, Japan) were performed under anesthesia (continuous intravenous infusion of propofol $0.2-0.6 \mathrm{mg} / \mathrm{kg} / \mathrm{min}$ ). Overlay MR and

CT images were created using PMOD® image analysis software (PMOD Technologies Ltd, Zurich, Switzerland) to estimate stereotaxic coordinates of target brain structures. After surgery, prophylactic antibiotics and analgesics 

amygdala ( $2 \mu \mathrm{L} \times 2$ locations). The monkey underwent a surgical procedure to open burr holes ( 8-mm diameter) for the injection needle. Viruses were pressureinjected using a 10- $\mu$ l Hamilton syringe (model 1701 RN, Hamilton) with a 30gauge injection needle and a fused silica capillary (Nagai et al., 2016). The Hamilton syringe was mounted into a motorized microinjector (UMP3T-2, WPI) that was held by a manipulator (model 1460, David Kopf) on the stereotaxic frame. After the dura mater was opened about $3 \mathrm{~mm}$, the injection needle was inserted into the brain and slowly moved down $2 \mathrm{~mm}$ beyond the target and then kept stationary for $5 \mathrm{~min}$, after which it was pulled up to the target location. The injection speed was set at $0.25 \mu \mathrm{l} / \mathrm{min}$. After each injection, the needle remained in situ for $15 \mathrm{~min}$ to minimize backflow along the needle.

Two monkeys (\#229 and \#245) received injections of AAV vector into the bilateral prefrontal cortex (Brodmann's area 46) as described previously (Nagai et 180 al., 2020; Oyama et al., 2021). The frontal cortex was exposed by removing a bone 181 flap and reflecting the dura mater. Handheld injections at an oblique angle to the 182 brain surface were made under visual guidance through an operating microscope 183 (Leica M220, Leica Microsystems GmbH, Wetzlar, Germany). Nine tracks were 184 injected in each hemisphere located at the caudal tip (1 track) and along the dorsal 185 (4 tracks) and ventral (4 tracks) bank of the principal sulcus. Viral vectors were 186 injected at 3 to $5 \mu \mathrm{L}$ per track depending on the depth. A total of 35 to $44 \mu \mathrm{L}$ of viral 187 aliquots was injected into each hemisphere. 
189 PET imaging

190 PET imaging was conducted as previously reported (Nagai et al., 2020). Briefly,

191 PET scans were performed using a microPET Focus 220 scanner (Siemens

192 Medical Solutions USA, Malvern, USA). Monkeys were immobilized by ketamine

$193(5-10 \mathrm{mg} / \mathrm{kg})$ and xylazine $(0.2-0.5 \mathrm{mg} / \mathrm{kg})$ and then maintained under anesthetized

194 condition with isoflurane (1-3\%) during all PET procedures. Transmission scans

195 were performed for about 20 min with a Ge-68 source. Emission scans were

196 acquired in 3D list mode with an energy window of 350-750 keV after intravenous

197 bolus injection of $\left[{ }^{11} \mathrm{C}\right] \mathrm{DCZ}$ (324.9-382.3 MBq). Emission data acquisition lasted for

$19890 \mathrm{~min}$. To estimate the specific binding of $\left[{ }^{11} \mathrm{C}\right] \mathrm{DCZ}$, regional binding potential

199 relative to nondisplaceable radioligand $\left(\mathrm{BP}_{\mathrm{ND}}\right)$ was calculated by $\mathrm{PMOD} \AA$ with an

200 original multilinear reference tissue model (MRTMo)(Yan et al., 2021). PET scans

201 were conducted at 45 days after injection of vectors for the two monkeys (\#229 and

202 \#245) and for one monkey expressing hM4Di in the amygdala (\#237) between 496

203 and 984 days, during which enough hM4Di expression for the occupancy study

204 was observed after its introduction.

205

206 Drug administration

207 DCZ (MedChemExpress HY-42110) was dissolved in 2.5\% dimethyl sulfoxide

208 (DMSO, FUJIFILM Wako Pure Chemical Co.), aliquoted and stored at $-30^{\circ} \mathrm{C}$. For

209 systemic i.m. injection, this stock solution was diluted in saline to a final volume of 
$210100 \mu \mathrm{g} / \mathrm{kg}$, and injected intramuscularly. For systemic oral administration (per os,

211 p.o.), the $D C Z$ stock solution was diluted in saline to a final volume of $100 \mu \mathrm{g} / \mathrm{kg}$,

$212300 \mu \mathrm{g} / \mathrm{kg}$, or $1,000 \mu \mathrm{g} / \mathrm{kg}$. The solution was then injected into some pieces of

213 snack or diluted in drinking water, which were given to the monkeys. It typically

214 took 2-3 min for the monkeys to consume. After each administration, we confirmed

215 that there was no food in the monkeys' mouths. Fresh solution was prepared on

216 each day of usage.

\section{Occupancy study}

219 An occupancy study was performed using one monkey expressing hM4Di in the 220 amygdala (\#237), because it is ideal for placing voxels of interest and measuring tracer binding in a large volume of tissue expressing hM4Di and non-DREADD control on the other side, as in previous studies (Nagai et al., 2016, 2020). PET procedures were described above except for pretreatment of DCZ or vehicle.

224 Pretreatment of DCZ or vehicle p.o. via a gastric catheter was followed by anesthesia and emission scans at 30 and 90 min, respectively. The baseline scan data were obtained within 3 weeks from the day of PET scans for occupancy measurement to minimize the effect of hM4Di expression level changes during this period. To estimate the specific binding of $\left[{ }^{11} \mathrm{C}\right] \mathrm{DCZ}, \mathrm{BP}_{\mathrm{ND}}$ was calculated by

229 PMOD® with MRTMo. The volume of interest for the hM4Di-positive region was defined as the area where the $\mathrm{BP}_{\mathrm{ND}}$ value of $\left[{ }^{11} \mathrm{C}\right] \mathrm{DCZ}$ was higher than 0.5 , while that of the control region was placed at the corresponding contralateral side. 
Estimates of fractional occupancy were calculated according to the previous 233 study (Nagai et al., 2020) by the following equation:

$$
O c c=\left({ }^{h M 4 D i} B P_{B L}-{ }^{h M 4 D i} B P_{P T}\right) /\left({ }^{h M 4 D i} B P_{B L}-{ }^{C O N} B P_{B L}\right)
$$

where ${ }^{h M 4 D i} B P_{B L}$ and ${ }^{C O N} B P_{B L}$ indicate $\mathrm{BP}_{\mathrm{ND}}$ at the $\mathrm{hM}$ Di-expressing amygdala region and contralateral control region under baseline conditions, respectively, while ${ }^{h M 4 D i} B P_{P T}$ indicates $\mathrm{BP}_{\mathrm{ND}}$ at the $\mathrm{hM}_{4}$ Di-expressing amygdala region under pretreatment condition. The relationship between occupancy (Occ) and agonist dose $\left(D_{D C Z}\right)$ was modeled by the Hill equation

$$
O C C=D_{D C Z}{ }^{n} /\left(D_{D C Z}{ }^{n}+E D_{50}{ }^{n}\right)
$$

where $E D_{50}$ and $n$ indicate the agonist dose achieving 50\% occupancy and the Hill 242 coefficient, respectively.

\section{Pharmacokinetics analysis}

245 Three monkeys (\#226, \#248, \#249) were used to assess the concentration of DCZ 246 and its metabolites in plasma or cerebrospinal fluid (CSF). First, the monkeys were 247 administered DCZ intramuscularly or orally (100 $\mu \mathrm{g} / \mathrm{kg}$ for i.m.; $300 \mu \mathrm{g} / \mathrm{kg}$ for p.o.).

248 Then they were immobilized by i.m. injection of ketamine $(5-10 \mathrm{mg} / \mathrm{kg})$ and xylazine $249(0.2-0.5 \mathrm{mg} / \mathrm{kg})$ and intubated with an endotracheal tube. Anesthesia was 250 maintained with isoflurane (1-3\%, to effect). Then blood and CSF were collected at $25130,60,90,120,150,180,210,240 \mathrm{~min}$ after DCZ administration, and on the next 252 day, monkeys were again anesthetized, and the samples were collected at $24 \mathrm{~h}$ 253 after DCZ administration. Blood was collected with a heparinized syringe and 
254 plasma samples were obtained by centrifugation at 3,500 $\mathrm{g}$ for $15 \mathrm{~min}$. All samples 255 were stocked at $-80^{\circ} \mathrm{C}$ until analysis.

256 The pretreatment protocols for CSF and plasma samples were described 257 previously (Nagai et al., 2020). Quantification of DCZ, and its metabolites C21 and 258 DCZ-N-oxide, was performed by multiple reaction monitoring (MRM) using a 259 Shimadzu UHPLC LC-30AD system (Shimadzu Corp., Kyoto, Japan) coupled to a 260 tandem MS AB Sciex Qtrap 6500 system (AB Sciex LLC, Framingham, MA, USA).

261 The following MRM transitions (Q1/Q3) were used to monitor each compound:

262 DCZ (293.0/236.0), C21 (279.0/193.0), DCZ-N-oxide (309.0/192.9). Other 263 protocols were described previously.

264 A blood sample was also collected from one monkey (\#245) following oral 265 administration of $300 \mu \mathrm{g} / \mathrm{kg}$ of DCZ along with the daily behavioral testing under 266 awake conditions to quantify the concentration of DCZ. The collected sample was 267 treated and analyzed as well as those described above.

268

\section{Behavioral task}

270 Three monkeys (\#226, \#229, \#245) were tested with a spatial delayed 271 response task. The protocol was almost the same as in our previous study (Oyama 272 et al., 2021). Behavioral testing was conducted in a sound-attenuated room.

273 Monkeys sat on a monkey chair from which they could reach out one hand and 274 take food to their mouths. A wooden table with two food-wells was placed in front of 275 the monkeys, and a screen was placed between the monkeys and the table. First, 
276 a piece of food reward (raisin or snack) was placed in one of the two food-wells,

277 and then both wells were covered with wooden plates. Then, the screen was

278 placed for $0.5,10$ or $30 \mathrm{~s}$, which served as delay periods. The position of the baited

279 well (left or right) and the delay period were determined pseudo-randomly. After the

280 delay period, the screen was removed and the monkeys were allowed to select

281 either food-well. The monkeys were allowed to get the food if they reached for the

282 correct food-well and removed the cover plate. The inter-trial interval was set at 10

283 s. A daily session lasted about one hour, and consisted of 3 blocks of 30 trials for

284 monkey \#229, and 2 blocks of 30 trials for monkeys \#226 and \#245, which were

285 interleaved with 5-min rest periods. Behavioral tests began immediately or $0.5,1,3$,

286 or $24 \mathrm{~h}$ after vehicle or DCZ treatment.

287 Another monkey without AAV injection (non-DREADD; \#239) was tested with

288 a reward-size task using the same protocol as applied in a previous study

289 (Minamimoto et al., 2009). The behavioral testing began $1 \mathrm{~h}$ after an oral

290 administration of either vehicle or DCZ.

291

292 Statistics

293 To examine the effect of each treatment on the performance of the delayed

294 response task, behavioral measurement (correct rates) was subjected to Welch's t-

295 test or two-way ANOVA using GraphPad Prism 7.

296

297 Histology and immunostaining 
298 For histological inspection, two monkeys (\#229, \#245) were deeply anesthetized

299 with an overdose of sodium pentobarbital ( $80 \mathrm{mg} / \mathrm{kg}$, i.v.) and transcardially

300 perfused with saline at $4^{\circ} \mathrm{C}$, followed by $4 \%$ paraformaldehyde in $0.1 \mathrm{M}$ phosphate

301 buffered saline (PBS), pH 7.4. The brain was removed from the skull, postfixed in

302 the same fresh fixative overnight, saturated with $30 \%$ sucrose in phosphate buffer

$303(\mathrm{~PB})$ at $4^{\circ} \mathrm{C}$, and then cut serially into $50-\mu \mathrm{m}$-thick sections on a freezing

304 microtome. For visualization of immunoreactive signals of GFP (co-expressed with

$305 \mathrm{hM} 4 \mathrm{Di})$, a series of every 6th section was immersed in $1 \%$ skim milk for $1 \mathrm{~h}$ at

306 room temperature and incubated overnight at $4^{\circ} \mathrm{C}$ with rabbit anti-GFP monoclonal

307 antibody (1:200-500, G10362, Thermo Fisher Scientific) in PBS containing 0.1\%

308 Triton $\mathrm{X}-100$ and $1 \%$ normal goat serum for 2 days at $4^{\circ} \mathrm{C}$. The sections were then

309 incubated in the same fresh medium containing biotinylated goat anti-rabbit lgG

310 antibody (1:1,000; Jackson ImmunoResearch, West Grove, PA, USA) for $2 \mathrm{~h}$ at

311 room temperature, followed by avidin-biotin-peroxidase complex (ABC Elite, Vector

312 Laboratories, Burlingame, CA, USA) for $2 \mathrm{~h}$ at room temperature. For visualization

313 of the antigen, the sections were reacted in $0.05 \mathrm{M}$ Tris- $\mathrm{HCl}$ buffer $(\mathrm{pH} 7.6)$

314 containing $0.04 \%$ diaminobenzidine (DAB), $0.04 \% \mathrm{NiCl} 2$, and $0.003 \% \mathrm{H}_{2} \mathrm{O}_{2}$. The

315 sections were mounted on gelatin-coated glass slides, air-dried, and cover-slipped.

316 A part of other sections was Nissl-stained with 1\% Cresyl violet. Images of sections

317 were digitally captured using an optical microscope equipped with a high-grade

318 charge-coupled device (CCD) camera (Biorevo, Keyence, Osaka, Japan). 


\section{Results}

\section{Pharmacokinetics of orally administered DCZ}

321 We first conducted pharmacokinetic studies in three naïve monkeys to estimate the 322 time course of the chemogenetic effects of DCZ via oral administration. In our 323 previous study, intramuscular administration of DCZ at $100 \mu \mathrm{g} / \mathrm{kg}$ provided a 324 sufficient level of DCZ concentration in CSF to activate hM4Di receptors for $2 \mathrm{~h}$, 325 during which it gave rise to behavioral deficits in monkeys via hM4Di activation 326 (Nagai et al., 2020). Given that oral administration of clozapine, an analogue of 327 DCZ, results in relatively lower bioavailability and slower onset of action compared 328 to intravenous administration (Sun and Lau, 2000), we first examined DCZ 329 concentrations in the plasma and CSF for $4 \mathrm{~h}$ following oral DCZ administration $330(300 \mu \mathrm{g} / \mathrm{kg})$, as well as intramuscularly $(100 \mu \mathrm{g} / \mathrm{kg})$ as reference. Oral DCZ 331 administration yielded a maximum DCZ concentration in plasma at 60 min after 332 administration, followed by a gradual decrease (Fig. 1A, orange). By contrast, 333 intramuscular administration increased plasma DCZ concentration at $30 \mathrm{~min}$, which 334 then decreased monotonically (Fig. 1A, green). Both oral and intramuscular 335 administrations produced relatively stable DCZ concentrations in CSF for at least 4 $336 \mathrm{~h}(>2 \mathrm{nM}$ and $>5 \mathrm{nM}$; Fig. 1B, orange and green, respectively), which were 337 sufficient concentrations to activate hM4Di DREADD ( $\mathrm{EC}_{50}$ of $0.081 \mathrm{nM}$ ) (Nagai et 338 al., 2020). DCZ was not detected in plasma or CSF $24 \mathrm{~h}$ after administration. We 339 also examined the concentration of possible metabolites of DCZ, C21 and DCZ-N340 oxide in plasma; however, metabolites were not detected (Fig. 1A, light blue and 
341 purple), confirming the metabolic stability of DCZ (Nagai et al., 2020). Taken 342 together, these results suggest that the oral administration of DCZ produces a 343 relatively long-lasting pharmacokinetic profile without significant metabolite 344 production and a lower bioavailability, about $10-20 \%$ of that of intramuscular 345 administration.

Measuring in vivo occupancy of hM4Di-DREADD by oral DCZ administration

348 Next, to determine an effective and safe dose range for oral DCZ administration, 349 we measured hM4Di receptor occupancy by DCZ - the relationship between DCZ 350 dose and the degree of hM4Di receptor occupation (Fig. 2A). This was important 351 because a higher agonist dose will afford a stronger chemogenetic effect, but may 352 risk higher off-target effects (Upright and Baxter, 2020). Normally, proportional 353 receptor occupancy is a generalizable measure because it reflects the relationship 354 between target drug concentration and its receptor affinity, irrespective of brain 355 region or receptor density(Cunningham et al., 2010; Takano et al., 2016). In our 356 previous study, a series of PET studies was used to determine the optimal DCZ 357 dose that would yield a $50-80 \%$ occupancy of hM4Di, which was $30-100 \mu \mathrm{g} / \mathrm{kg}$ for 358 systemic administration (Fig. 2C, see Nagai et al., 2020 for details). Given that the 359 bioavailability of oral administration would be $10-20 \%$ of that of systemic 360 administration (Fig. 1), we estimated that oral DCZ doses achieving $50 \%$ and $80 \%$ $361 \mathrm{hM} 4 \mathrm{Di}$ occupancy would be around 300 and $1,000 \mu \mathrm{g} / \mathrm{kg}$, respectively. We 
362 performed a PET occupancy study using a monkey that had received injection of a 363 viral vector encoding an hM4Di gene into the unilateral amygdala (see Methods). 364 Baseline PET imaging with radiolabeled DCZ $\left(\left[{ }^{11} \mathrm{C}\right]-\mathrm{DCZ}\right)$ confirmed that the 365 hM4Di expression at the injected side of the amygdala was indicated by an 366 increased radioligand binding (Fig 2B, baseline). Subsequent $\left[{ }^{11} \mathrm{C}\right]-\mathrm{DCZ}$ PET scans 367 were performed after oral pretreatments (i.e., oral administration of vehicle, 100, 368300 , or $1,000 \mu \mathrm{g} / \mathrm{kg}$ of non-radiolabeled DCZ). Consistent with our previous 369 findings, specific binding of $\left[{ }^{11} \mathrm{C}\right] \mathrm{DCZ}$ at the hM4Di-expressing amygdala region 370 was diminished with increasing doses of DCZ (Fig. 2B). Occupancy was 371 determined as a reduction of specific radioligand binding $\left(\mathrm{BP}_{\mathrm{ND}}\right)$ at the target 372 region over the control side relative to baseline (Fig. 2B, see Materials \& Methods 373 for details). As we predicted, the hM4Di occupancies with 100, 300 and 1,000 $374 \mu \mathrm{g} / \mathrm{kg}$ oral administration were about $35 \%, 50 \%$ and $70 \%$, respectively (Fig. 2C).

375 The dose for $50 \%$ occupancy $\left(E_{50}\right)$ was $254 \mu \mathrm{g} / \mathrm{kg}$, which corresponded to about 376 a 10-fold dose of systemic administration and was roughly consistent with the 377 bioavailable profile shown in our pharmacokinetic study described above. Based 378 on these results in pharmacokinetic and occupancy studies, we determined the 379 effective and safety dose range for oral DCZ administration to be $300-1,000 \mu \mathrm{g} / \mathrm{kg}$. 380 381 Oral DCZ administration selectively induces longer-lasting behavioral effects 382 in hM4Di-expressing monkeys 
383

384

385

386

387

388

389

390

391

392

393

394

395

396

397

398

399

400

401

402

403

404

We next examined the efficacy and time-course of the chemogenetic effect of oral DCZ administration using hM4Di-expressing monkeys. We used two monkeys with hM4Di expressed in the bilateral dorsolateral prefrontal cortex (dIPFC) around the principal sulcus (Brodmann's area 46; Fig. 3A,B), a brain region known to be responsible for spatial working memory and executive function (Fuster, 2015). Several weeks after the injections, we performed PET imaging with $\left[{ }^{11} \mathrm{C}\right] \mathrm{DCZ}$ to visualize hM4Di expression non-invasively. High levels of $\left[{ }^{11} \mathrm{C}\right] \mathrm{DCZ}$ binding $\left(\mathrm{BP}_{\mathrm{ND}}>\right.$ 1.0) were localized in the bilateral dIPFC in both monkeys, specifically from the caudal end of the principal sulcus to 5-6 mm anterior to it (Fig. 3C,F). These PET signals reflected hM4Di expression, as post-mortem analysis confirmed GFPpositive zone in dorsal and ventral bank of the principal sulcus (Fig. 3D-H). When we compared the time course of $\left[{ }^{11} \mathrm{C}\right] \mathrm{DCZ}$ uptake in the whole brain of these monkeys with that of other monkeys that had not undergone surgery (Fig. 3I), there was no marked difference across the subjects. This suggested that the brain permeability of DCZ was unaffected by the dIPFC surgery.

Our previous studies have shown that chemogenetic silencing of the dIPFC via intramuscular DCZ administration $(100 \mu \mathrm{g} / \mathrm{kg})$ in these monkeys impaired performance in the spatial delayed response task (Fig. 4A) (Nagai et al., 2020;

Oyama et al., 2021). Prior to the introduction of hM4Di, intramuscular DCZ administration $(100 \mu \mathrm{g} / \mathrm{kg})$ per se did not have an impact on working memory in \#245 (Fig. 4B; Nagai et al., 2020), suggesting that oral administration of DCZ below $1,000 \mu \mathrm{g} / \mathrm{kg}$ would be expected not to have any side-effects. Then we directly 
compared the effects of oral administration with those of intramuscular injections by using the same subjects as in our previous studies that showed similar behevioaral deficits (Nagai et al., 2020), which would enable us to demonstrate that oral DCZ administration can be adapted to dIPFC, one of the core brain regions specialized for primates. We examined the behavioral effects of two oral DCZ doses (300 and $1,000 \mu \mathrm{g} / \mathrm{kg})$ and an intramuscular DCZ dose $(100 \mu \mathrm{g} / \mathrm{kg})$ for comparison. Consistent with previous studies (Nagai et al., 2020; Oyama et al., 2021), intramuscular $100 \mu \mathrm{g} / \mathrm{kg} \mathrm{DCZ}$ administration resulted in a delay-dependent decrease in the task performance for 1-2 $\mathrm{h}$ following administration (Fig. 4E). Oral DCZ administration of 300 and 1,000 $\mu \mathrm{g} / \mathrm{kg} \mathrm{DCZ}$ also induced a comparable impairment of the task performance for 1-2 $\mathrm{h}$ after administration (Figs. $4 \mathrm{C}$ and $\mathrm{D}$ ). We further assessed the time-course of the chemogenetic effects by conducting a behavioral examination at 5 different time windows after treatment: $0-0.5,0.5-1$, $1-2,3-4$, and $24-25 \mathrm{~h}$. We focused on the performance of the trials with the longest delay period, where the effects were most prominent (Figs. 4C-E, 30s). In the first 30-min period, intramuscular administration significantly decreased the correct rates (Fig. 4H, green) compared to those after vehicle administration (Fig. $4 \mathrm{H}$, cyan), indicating rapid behavioral effects as previously demonstrated (Nagai et al., 2020). Significant effects were also observed following oral administration of 300 and 1,000 $\mu \mathrm{g} / \mathrm{kg}$ (Figs. 4F, G, orange and magenta, respectively). Similarly, all treatments had significant effects at $0.5-1$ and $1-2 \mathrm{~h}$ after administration (Fig. 4FH). At 3-4 h after administration, intramuscular administration had no influence on 
427 performance (Fig. $4 \mathrm{H}$ ), while the oral administrations remained effective (Figs. 4F, 428 G).

To determine the minimum effective dose of DCZ via oral administration, we 430 also administered $100 \mu \mathrm{g} / \mathrm{kg} \mathrm{DCZ}$ orally and examined the monkeys $1-2$ and $3-4 \mathrm{~h}$ 431 thereafter. The performance was significantly impaired after 1-2 $\mathrm{h}$ (Welch's t-test, $\mathrm{t}$ $432=4.2, p=0.002)$, but not after $3-4 \mathrm{~h}(\mathrm{t}=0.48, \mathrm{p}=0.65)$, indicating that this dose is 433 effective only for a shorter duration compared to 300 and $1,000 \mu \mathrm{g} / \mathrm{kg}$. Importantly, the decrease in task performance was found to be dependent on delay duration (Fig. 4C), suggesting that the orally administered DCZ resulted in 436 loss of dIPFC function, but did not produce side-effects on other functions, such as general attention or motivation. To further confirm that oral administration had no unwanted side effects, we conducted control experiments with the delayed response task (Fig. 5A) and a simple instrumental task (Fig. 5B, C) in two monkeys

440 to whom DREADDs had not been introduced (non-DREADD monkeys). Similar to 441 our previous study that tested with $100 \mu \mathrm{g} / \mathrm{kg}$ of intramuscular DCZ administration 442 (Nagai et al., 2020), oral DCZ administration of $1,000 \mu \mathrm{g} / \mathrm{kg}$ did not produce any 443 discernible behavioral changes in either task (Fig. 5A, C), suggesting that oral DCZ 444 administration itself is behaviorally inert at a dose of $1,000 \mu \mathrm{g} / \mathrm{kg}$ or less. Together, these results suggest that oral DCZ administration can induce 446 behavioral effects selectively in hM4Di-expressing monkeys for hours without 447 adverse side effects; specifically, a dose of $300-1,000 \mu \mathrm{g} / \mathrm{kg}$ is effective for at least 448 $4 \mathrm{~h}$. 
450 Chronic DCZ administration enables successive induction of chemogenetic 451 silencing for weeks

452 Finally, we investigated the efficacy of chronic DCZ administration across weeks.

453 One monkey expressing hM4Di in the dIPFC was tested daily with the delayed

454 response task to examine the chemogenetic effect of repetitive oral DCZ

455 administration. Chronic oral DCZ administrations for at least 2 weeks were

456 preceded and followed by several days of control vehicle administration (Fig. 6A,

457 B), and repeated twice with a sufficient interval of several months. This design

458 allowed us to investigate whether repetitive DCZ dose would consistently induce

459 behavioral deficits for weeks and how soon task performance would be recovered

460 after withdrawal of DCZ. Daily oral DCZ administrations $(300 \mu \mathrm{g} / \mathrm{kg})$ consistently

461 induced impairment of the performance of the delayed response task during $1-2 \mathrm{~h}$

462 post-administration compared to that of following vehicle injections throughout the

463 repetitive oral DCZ period (Fig. 6C), indicating that apparent desensitization did not

464 occur. Previous studies have shown that chronic chemogenetic manipulation

465 caused posttreatment effects, such as rebound excitability (Desloovere et al., 2019;

466 Goossens et al., 2021), suggesting that it could induce some physiological

467 changes lasting even after agonist withdrawal. That was not the case in the current

468 study: we found that performance recovered to a normal level on the first day after

469 withdrawing from chronic DCZ administration (Fig. 6C), indicating that successive 
470 behavioral deficits were the outcome of temporal chemogenetic silencing rather

471 than due to physiological change such as DREADD-induced plasticity.

472 It has been shown that plasma concentrations of the DCZ analogue

473 clozapine vary within subjects over weeks of treatment (Centorrino et al., 1994),

474 especially dropping by an average of more than $50 \%$ in the first 12 weeks (Kurz et

475 al., 1998). We confirmed that the concentration of DCZ following daily

476 administration was maintained at a similar level throughout the period of DCZ

477 administration, indicating that chronic manipulation did not alter the

478 pharmacokinetics of DCZ for at least 2 weeks (Fig. 6D). We also verified that

479 chronic DCZ administration for a single schedule (16 days for DCZ, and 7 and 3

480 days of vehicle administration, see Fig. 6A) alone had no significant impact on

481 spontaneous movements in a non-DREADD monkey (\#253; Welch's t-test, $\mathrm{t}=$

$4820.55, p=0.59)$. Taken together, these results suggested that $D C Z$ is suitable for a

483 chronic and reversible manipulation of neuronal activity, leading to potential

484 therapeutic applications in the future.

485

486 


\section{Discussion}

Here we demonstrated that oral administration of DCZ is an effective and noninvasive means for manipulating neuronal activity through hM4Di receptors in macaque monkeys. Our pharmacokinetics and in vivo PET occupancy examination showed that the bioavailability of orally administered DCZ was about $10-20 \%$ of that of intramuscular injection, and thus the appropriate dosage of oral DCZ to exert hM4Di-mediated chemogenetic effects was estimated to be $300-1,000 \mu \mathrm{g} / \mathrm{kg}$. Indeed, this oral DCZ at this range of doses caused severe impairments in working memory performance for several hours in monkeys with hM4Di expressed in dIPFC, but did not induce any discernible behavioral side-effects in control nonDREADD-expressing monkeys. Furthermore, repetitive daily oral DCZ doses over two weeks yielded consistent chemogenetic effects without apparent signs of desensitization. Taken together, the oral administration of DCZ affords a minimally invasive strategy for chronic and reversible chemogenetic control of neuronal activity via muscarinic DREADDs in nonhuman primates, thereby providing a great potential for its clinical application.

For DREADDs studies in rodents and nonhuman primates, systemic (intraperitoneal, intramuscular, or intravenous) injections are the standard approach of agonist administration, as they allow precise control of dosage and timing. However, such approach forces the animals to be restrained, which may cause stress responses and unwanted effects on behavioral actions. Several DREADDs studies have demonstrated that oral administration seems effective for 
overcoming this issue. For example, oral $\mathrm{CNO}$ administration induced changes in ethanol consumption by inhibition of the nucleus accumbens via hM4Di, in which neuronal activity was remotely manipulated while the animals were unrestrained (Cassataro et al., 2014). This is beneficial when wanting to examine the effect on natural behavior including social communication (Zou et al., 2016). Oral CNO administration has also been applied to manipulate the activity of non-neuronal cells such as microglia (Grace et al., 2016). However, recent reports have revealed that the effects of CNO are partly mediated by its back-conversion to clozapine which crosses the blood-brain barrier and acts as a DREADDs actuator (Gomez et al., 2017). Oral administration of low doses of clozapine and olanzapine as agonists has also been attempted (Goossens et al., 2021). Due to their high affinities for endogenous receptors, however, possible side effects are always a matter of concern that cannot completely be ruled out.

In the current study, we evaded these issues by using DCZ, a novel DREADDs agonist with high brain permeability and high selectivity. Since the effects of drugs on the central nervous system depend on multiple factors such as brain permeability, drug kinetics, and affinity for target receptors, it is generally difficult to predict the optimal drug dosage. As we have demonstrated in previous studies (Nagai et al., 2016, 2020), PET occupancy measurements together with pharmacokinetics analysis are extremely effective ways for seeking the adequate agonist dose range. We found that the bioavailability of orally administered DCZ was $10-20 \%$ compared to systemic injections (see Figs. 1 and 2). Based on this 
531 estimation, the dose range suitable for hM4Di activation was determined to be $300-$

$5321,000 \mu \mathrm{g} / \mathrm{kg}$. In line with the kinetics data, such oral DCZ doses induced relatively 533 long-lasting effects compared to intramuscular injections. The DCZ kinetics and 534 behavioral effects were examined up to $4 \mathrm{~h}$ after oral administration, during which 535 the concentration of DCZ persisted in CSF, and thus the chemogenetic effects may 536 be maintained longer.

537 The working-memory impairment following DCZ administration cannot be 538 explained by side-effects due to action on endogenous receptors as indicated by 539 other prior DREADDs agonists for the following reasons. First, one of the two 540 monkeys (\#245) had been tested with the delayed response task before 541 introducing hM4Di in the dIPFC, showing that DCZ alone had no impact on the 542 behavior (Fig. 4B). Second, control experiments using non-DREADD expressing 543 monkeys confirmed that these oral DCZ doses alone did not produce any 544 discernible changes in behavior associated with spatial working memory or reward 545 expectation (Fig. 5). Third, PET and histological data suggest that two monkeys 546 consistently express hM4Di, covering the similar region of dIPFC. Collectively, we 547 concluded that behavioral results consistently observed in these two monkeys were 548 due to the inactivation of dIPFC neurons via hM4Di activation. In the present study, we did not examine the effects of oral DCZ 550 administration on $\mathrm{hM} 3 \mathrm{Dq}$, an excitatory muscarinic DREADDs that responds to 551 DCZ (Nagai et al., 2020). Our previous work showed that the lower doses (1-3 $552 \mu \mathrm{g} / \mathrm{kg}$ ) of DCZ through intramuscular injection were capable of inducing significant 
553 neuronal excitation in mice and monkeys expressing hM3Dq (Nagai et al., 2020).

554 Thus, 3-30 $\mu \mathrm{g} / \mathrm{kg}$ would be an effective range for activating hM3Dq via oral

555 administration. Indeed, we have recently demonstrated that marmosets expressing

$556 \mathrm{hM}$ Dq produced consistent behavioral changes when fed a diet containing 10

$557 \mu \mathrm{g} / \mathrm{kg}$ of DCZ (Mimura et al., 2021). In any case, it should be noted that the optimal

558 agonist dose generally depends on the level of the overexpressed functional

559 protein (Upright et al., 2018) and the targeted circuit to be manipulated. In addition,

560 as is usual in studies using genetic methods, control experiments (e.g., using non-

561 DREADDs animals) are recommended.

562 It has been shown that chronic administration of DREADDs agonists

563 induces DREADDs-mediated changes for days to weeks in rodents (Nation et al.,

564 2016; Desloovere et al., 2019; Paretkar and Dimitrov, 2019; Goossens et al.,

565 2021). However, some studies reported that the effects following administrations of

566 agonists were not consistent during treatments. For example, Goossens et al.

567 reported that the administrations of clozapine and olanzapine caused significant

568 behavioral changes for several days, but that such effects subsequently diminished

569 even though the agonist administrations continued (Goossens et al., 2021). In

570 addition, it has been reported that inhibition by hM4Di with agonist treatment over

571 weeks induced posttreatment rebound excitability (Desloovere et al., 2019;

572 Goossens et al., 2021). In contrast to these studies, we showed that daily oral DCZ

573 delivery constantly impaired the monkey's performance throughout the

574 administration periods (see Fig. 6). Moreover, performance on the day following 
completion of repeated doses of DCZ was as high as the baseline, suggesting that weeks-long inactivation of the PFC did not affect subsequent behavior. These results suggest that chronic $\mathrm{DCZ}$ administration did not cause desensitization of hM4Di or any long-lasting change, such as plasticity of neural circuits that govern working memory. It remains to be clarified whether this is a general capability of DREADD/DCZ or is due to the characteristics of neurons and local circuits in the dIPFC as a target. Irrespective of the mechanism, the present results indicate that it is possible to investigate the effects of chronic attenuation of PFC activity on various cognitive functions. Importantly, it can resolve potential discrepancies that may arise in the behavioral outcomes of different durations or methods of inactivation. For example, it has been demonstrated that acute and chronic silencing of PFC interneurons had opposite impacts on anxiety-like behavior in mice (Soumier and Sibille, 2014), suggesting that short- vs. long-term manipulations of local circuits may differentially alter PFC network functions. Besides, long-term abnormalities or imbalances in prefrontal activity have been suggested as a pathophysiological mechanism for psychiatric disorders, since chronic stress exposure could lead to anxiety disorder and depression (Zhou et al., 2019). DREADDs subserve to mimic or reverse such long-term circuitry changes that are beyond the reach of conventional acute blockade and/or irreversible lesioning. The chemogenetic approach using DCZ as an actuator introduced in the present study will expand the opportunity of acute and chronic manipulations of specific circuits in the primate prefrontal cortex, thereby leading to a better 
597 understanding of higher brain functions, e.g., working memory, decision-making,

598 and action-planning, in health and disease conditions. This can be done without 599 causing pain/stress to monkeys, and thus is also suitable for quantifying the effects

600 on unrestrained naturalistic, social behavior (e.g., using a markerless motion

601 tracking system; Bala et al., 2020; Labuguen et al., 2021). In addition, it has the

602 potential to be used in therapeutic applications for symptoms caused by abnormal

603 neuronal activity in a specific brain region, such as seizures in epilepsy.

604 Finally, it should be noted that this study did not examine continuous

605 chemogenetic effects beyond the duration of action of a single oral dose $(\sim 4 \mathrm{~h})$.

606 Continuous silencing may be achieved with more frequent and repeated

607 administrations. It should also be noted that the utility of chronic oral administration

608 and the effect of DCZ in pre-DREADD condition were examined in a single monkey

609 (\#245). Future studies will need to determine the dosage and interval of

610 administrations to maintain sufficient DCZ concentration to attain continuous

611 chemogenetic effects, and to ensure that it does not cause undesirable side

612 effects, toxicity or tachyphylaxis, with appropriate within-subject control using non-

613 DREADD animals.

614 DREADDs technology is becoming increasingly popular as a means of 615 controlling neuronal activity remotely, less-invasively and reproducibly in rodents 616 and nonhuman primates. With the accumulation of numerous successful reports, 617 muscarinic DREADDs are now under consideration for clinical application. Given 618 the long-term stable chemogenetic effects with orally delivered DCZ in macaque 
619 monkeys, it holds great promise for the translational use of DREADDs technology,

620 especially in the development of therapeutic trials for neurological and

621 neuropsychiatric disorders.

622 
623

624

625

626

627

628

629

630

631

632

633

634

635

636

637

638

639

640

641

642

643

644

\section{References}

Andreoli M, Marketkar T, Dimitrov E (2017) Contribution of amygdala CRF neurons to chronic pain. Experimental neurology 298:1-12.

Bala PC, Eisenreich BR, Yoo SBM, Hayden BY, Park HS, Zimmermann J (2020) Automated markerless pose estimation in freely moving macaques with OpenMonkeyStudio. Nature Communications 11:4560.

Bonaventura J et al. (2019) High-potency ligands for DREADD imaging and activation in rodents and monkeys. Nature Communications 10:4627.

Cassataro D, Bergfeldt D, Malekian C, Van Snellenberg JX, Thanos PK, Fishell G, Sjulson L (2014) Reverse pharmacogenetic modulation of the nucleus accumbens reduces ethanol consumption in a limited access paradigm. Neuropsychopharmacology 39:283-290.

Centorrino F, Baldessarini RJ, Kando JC, Frankenburg FR, Volpicelli SA, Flood JG (1994) Clozapine and metabolites: concentrations in serum and clinical findings during treatment of chronically psychotic patients. Journal of clinical psychopharmacology 14:119-125.

Cunningham VJ, Rabiner EA, Slifstein M, Laruelle M, Gunn RN (2010) Measuring drug occupancy in the absence of a reference region: the Lassen plot revisited. Journal of cerebral blood flow and metabolism : official journal of the International Society of Cerebral Blood Flow and Metabolism 30:46-50.

Desloovere J, Boon P, Larsen LE, Merckx C, Goossens MG, Van den Haute C, Baekelandt V, De Bundel D, Carrette E, Delbeke J, Meurs A, Vonck K, 
Wadman W, Raedt R (2019) Long-term chemogenetic suppression of spontaneous seizures in a mouse model for temporal lobe epilepsy. Epilepsia 60:2314-2324.

Fuster JM (2015) The prefrontal cortex. Boston: Academic Press.

Gomez JL, Bonaventura J, Lesniak W, Mathews WB, Sysa-Shah P, Rodriguez LA, Ellis RJ, Richie CT, Harvey BK, Dannals RF, Pomper MG, Bonci A, Michaelides M (2017) Chemogenetics revealed: DREADD occupancy and activation via converted clozapine. Science 357:503-507.

Goossens M, Boon P, Wadman W, van den Haute C, Baekelandt V, Verstraete AG, Vonck K, Larsen LE, Sprengers M, Carrette E, Desloovere J, Meurs A, Delbeke J, Vanhove C, Raedt R (2021) Long - term chemogenetic suppression of seizures in a multifocal rat model of temporal lobe epilepsy. Epilepsia 62:659-670.

Grace PM, Strand KA, Galer EL, Urban DJ, Wang X, Baratta M V., Fabisiak TJ, Anderson ND, Cheng K, Greene LI, Berkelhammer D, Zhang Y, Ellis AL, Yin HH, Campeau S, Ricei KC, Roth BL, Maier SF, Watkins LR (2016) Morphine paradoxically prolongs neuropathic pain in rats by amplifying spinal NLRP3 inflammasome activation. Proceedings of the National Academy of Sciences of the United States of America 113:E3441-E3450.

Hirabayashi T, Nagai Y, Hori Y, Inoue K, Aoki I, Takada M, Suhara T, Higuchi M, Minamimoto T (2021) Chemogenetic sensory fMRI reveals behaviorally 
666

667

668

669

670

671

672

673

674

675

676

677

678

679

680

681

682

683

684

685

686

687

relevant bidirectional changes in primate somatosensory network. Neuron 109:3312-3322.e5.

Hori Y, Mimura K, Nagai Y, Fujimoto A, Oyama K, Kikuchi E, Inoue K, Takada M, Suhara T, Richmond BJ, Minamimoto T (2021) Single caudate neurons encode temporally discounted value for formulating motivation for action Izquierdo A, Behrens TE, Procyk E, Monosov IE, eds. eLife 10:e61248.

Karbwang J, Na-Bangchang K, Congpuong K, Molunto P, Thanavibul A (1997) Pharmacokinetics and bioavailability of oral and intramuscular artemether. European journal of clinical pharmacology 52:307-310.

Krause WC, Rodriguez R, Gegenhuber B, Matharu N, Rodriguez AN, PadillaRoger AM, Toma K, Herber CB, Correa SM, Duan X, Ahituv N, Tollkuhn J, Ingraham HA (2021) Oestrogen engages brain MC4R signalling to drive physical activity in female mice. Nature 599:131-135.

Kurz M, Hummer M, Kemmler G, Kurzthaler I, Saria A, Fleischhacker WW (1998) Long-term pharmacokinetics of clozapine. The British journal of psychiatry : the journal of mental science 173:341-344.

Labuguen R, Matsumoto J, Negrete SB, Nishimaru H, Nishijo H, Takada M, Go Y, Inoue KI, Shibata T (2021) MacaquePose: A Novel "In the Wild" Macaque Monkey Pose Dataset for Markerless Motion Capture. Frontiers in Behavioral Neuroscience 14:581154.

Lieb A, Weston M, Kullmann DM (2019) Designer receptor technology for the treatment of epilepsy. EBioMedicine 43:641-649. 
688

689

690

691

692

693

694

695

696

697

698

699

700

701

702

703

704

705

706

707

Mimura K, Nagai Y, Inoue K, Matsumoto J, Hori Y, Sato C, Kimura K, Okauchi T, Hirabayashi T, Nishijo H, Yahata N, Takada M, Suhara T, Higuchi M, Minamimoto T (2021) Chemogenetic activation of nigrostriatal dopamine neurons in freely moving common marmosets. iScience 24:103066.

Minamimoto T, La Camera G, Richmond BJ (2009) Measuring and modeling the interaction among reward size, delay to reward, and satiation level on motivation in monkeys. Journal of neurophysiology 101:437-447.

Nagai $Y$ et al. (2016) PET imaging-guided chemogenetic silencing reveals a critical role of primate rostromedial caudate in reward evaluation. Nature communications 7:13605.

Nagai Y et al. (2020) Deschloroclozapine, a potent and selective chemogenetic actuator enables rapid neuronal and behavioral modulations in mice and monkeys. Nature Neuroscience 23:1157-1167.

Nation HL, Nicoleau M, Kinsman BJ, Browning KN, Stocker SD (2016) DREADDinduced activation of subfornical organ neurons stimulates thirst and salt appetite. Journal of Neurophysiology 115:3123-3129.

Oyama K, Hori Y, Nagai Y, Miyakawa N, Mimura K, Hirabayashi T, Inoue K, Suhara T, Takada M, Higuchi M, Minamimoto T (2021) Chemogenetic dissection of the primate prefronto-subcortical pathways for working memory and decision-making. Science Advances 7:eabg4246. 
Paretkar T, Dimitrov E (2019) Activation of enkephalinergic (Enk) interneurons in the central amygdala $(\mathrm{CeA})$ buffers the behavioral effects of persistent pain. Neurobiology of Disease 124:364-372.

Roth BL (2016) DREADDs for Neuroscientists. Neuron 89:683-694.

Soumier A, Sibille E (2014) Opposing effects of acute versus chronic blockade of frontal cortex somatostatin-positive inhibitory neurons on behavioral emotionality in mice. Neuropsychopharmacology 39:2252-2262.

Sun L, Lau CE (2000) Intravenous and oral clozapine pharmacokinetics, pharmacodynamics, and concentration-effect relations: acute tolerance. European journal of pharmacology 398:225-238.

Takano A, Varrone A, Gulyás B, Salvadori P, Gee A, Windhorst A, Vercouillie J, Bormans G, Lammertsma AA, Halldin C (2016) Guidelines to PET measurements of the target occupancy in the brain for drug development. European Journal of Nuclear Medicine and Molecular Imaging 43:2255-2262.

Tran FH, Spears SL, Ahn KJ, Eisch AJ, Yun S (2020) Does chronic systemic injection of the DREADD agonists clozapine-N-oxide or Compound 21 change behavior relevant to locomotion, exploration, anxiety, and depression in male non-DREADD-expressing mice? Neuroscience Letters 739:135432.

Upright NA, Baxter MG (2020) Effect of chemogenetic actuator drugs on prefrontal cortex-dependent working memory in nonhuman primates. Neuropsychopharmacology 45:1793-1798. 
729

730

731

732

733

734

735

736

737

738

739

740

741

742

743

744

745

746

747

748

749

Upright NA, Brookshire SW, Schnebelen W, Damatac CG, Hof PR, Browning PGF, Croxson PL, Rudebeck PH, Baxter MG (2018) Behavioral effect of chemogenetic inhibition is directly related to receptor transduction levels in Rhesus monkeys. Journal of Neuroscience 38:7969-7975.

Urban DJ, Roth BL (2015) DREADDs (designer receptors exclusively activated by designer drugs): chemogenetic tools with therapeutic utility. Annu Rev Pharmacol Toxicol 55:399-417.

Wakaizumi K, Kondo T, Hamada Y, Narita M, Kawabe R, Narita H, Watanabe M, Kato S, Senba E, Kobayashi K, Kuzumaki N, Yamanaka A, Morisaki H, Narita M (2016) Involvement of mesolimbic dopaminergic network in neuropathic pain relief by treadmill exercise: A study for specific neural control with Gi-DREADD in mice. Molecular Pain 12:1-11.

Weston M, Kaserer T, Wu A, Mouravlev A, Carpenter JC, Snowball A, Knauss S, von Schimmelmann M, During MJ, Lignani G, Schorge S, Young D, Kullmann DM, Lieb A (2019) Olanzapine: A potent agonist at the hM4D(Gi) DREADD amenable to clinical translation of chemogenetics. Science Advances 5:eaaw1567.

Yan X, Telu S, Dick RM, Liow J-S, Zanotti-Fregonara P, Morse CL, Manly LS, Gladding RL, Shrestha S, Lerchner W, Nagai Y, Minamimoto T, Zoghbi SS, Innis RB, Pike VW, Richmond BJ, Eldridge MAG (2021) [11C]deschloroclozapine is an improved PET radioligand for quantifying a 
750 human muscarinic DREADD expressed in monkey brain. Journal of Cerebral $751 \quad$ Blood Flow \& Metabolism 41:2571-2582.

752 Yu S, Münzberg H (2018) Testing Effects of Chronic Chemogenetic Neuronal

753 Stimulation on Energy Balance by Indirect Calorimetry. Bio-protocol 8:e2811.

754 Zhou X-T, Bao W-D, Liu D, Zhu L-Q (2019) Targeting the Neuronal Activity of

755 Prefrontal Cortex: New Directions for the Therapy of Depression. Current

$756 \quad$ Neuropharmacology 18:332-346.

757 Zou D, Chen L, Deng D, Jiang D, Dong F, McSweeney C, Zhou Y, Liu L, Chen G, 758 Wu Y, Mao Y (2016) DREADD in parvalbumin interneurons of the dentate 759 gyrus modulates anxiety, social interaction and memory extinction. Current 760 molecular medicine 16:91-102.

761

762

763 


\section{Figure Legends}

765

766 Figure 1. Time concentration profiles of DCZ by oral and intramuscular

767 administration. A, Time-course of DCZ concentration in plasma (A) and CSF (B)

768 following intramuscular injection of $100 \mu \mathrm{g} / \mathrm{kg}$ (green) and oral administration of

$769300 \mu \mathrm{g} / \mathrm{kg}$ (orange) DCZ. Concentration of major metabolites of DCZ (DCZ-n-oxide

770 and C21, light blue and purple, respectively) following oral administration of 300

$771 \mu \mathrm{g} / \mathrm{kg} \mathrm{DCZ}$ are also shown (data for C21 were behind those of DCZ-n-oxide as

772 neither were detected). Data were collected from 3 monkeys (\#226, \#248, and

773 \#249). Error bars represent s.e.m.

774 
775 Figure 2. PET visualizes the occupancy of orally administered DCZ for hM4Di.

776 A, Schematic illustrations of occupancy study by PET. Monkeys underwent

$777 \quad\left[{ }^{11} \mathrm{C}\right] \mathrm{DCZ}-\mathrm{PET}$ scan $90 \mathrm{~min}$ after p.o. administration of nonradiolabeled DCZ or

778 vehicle. B, Coronal sections of parametric $\left[{ }^{11} \mathrm{C}\right] \mathrm{DCZ}-\mathrm{PET}$ image of specific binding

$779\left(\mathrm{BP}_{\mathrm{ND}}\right)$ overlaying MR image of a monkey expressing hM4Di in the amygdala.

780 Scale bars represent $10 \mathrm{~mm}$. C, Occupancy of hM4Di plotted as a function of DCZ.

781 Red triangles represent occupancy by oral DCZ doses obtained from monkey

782 \#237. Curves are the best-fit Hill equation for the data. $E D_{50}$ indicates the agonist

783 dose inducing $50 \%$ occupancy. Hill coefficient (n) and coefficient of determination

$784\left(R^{2}\right)$ values are as follows: p.o. $n=0.66, R^{2}=0.988$; i.v.: $n=1, R^{2}=0.99$. Note that

785 the data for intravenous (i.v.) injection (gray) refers to a previous study (Nagai et

786 al., 2020).

787 


\section{Figure 3. Introduction of hM4Di in bilateral dIPFC.}

789 A, Illustration showing the injection sites for AAV vector carrying an hM4Di gene.

790 The left panel presents a zoomed-in view of the framed area of the right panel. B,

791 A coronal plane corresponding to the lines in the lateral view. Red dots and shaded

792 area represent intended injection sites. $\mathbf{C , F}$, In vivo visualization of hM4Di

793 expression in dIPFC for \#229 (C) and \#245 (F). Coronal PET image showing

794 specific binding of $\left[{ }^{11} \mathrm{C}\right] \mathrm{DCZ}$ overlaying MR images. D,G, Corresponding nissl-

795 stained sections for \#229 (D) and \#245 (G). E,H, Corresponding DAB-stained

796 sections representing immunoreactivity against a reporter protein for \#229 (E) and

797 \#245 (H). Open and filled arrowheads represent the dorsal and ventral borders of

798 the target regions, respectively. Scale bars, $5 \mathrm{~mm}$. I, The time course of whole-brain

799 uptake of $\left[{ }^{11} \mathrm{C}\right] \mathrm{DCZ}$ for 7 monkeys including \#229 and \#245. Shaded area

800 represents the standard deviation of 5 monkeys excluding \#229 and \#245. Please

801 note that \#229 and \#245 were the same subjects as used in our previous study

802 (Oyama et al., 2021). SUV, standardized uptake value [regional radioactivity

$803\left(\mathrm{~Bq} \mathrm{~cm}^{-3}\right) \times$ body weight $(\mathrm{g}) /$ injected radioactivity $\left.(\mathrm{Bq})\right]$. 


\section{Figure 4. Effects of oral and intramuscular administration of DCZ on a}

\section{5 cognitive task in monkeys expressing hM4Di in the dIPFC.}

806 A, Delayed response task. B, Effects of intramuscular administration of $100 \mu \mathrm{g} / \mathrm{kg}$

807 of DCZ on task performance for monkey \#245 before ("Pre-vector") and after

808 ("Post-vector") introduction of hM4Di (Nagai et al., 2020). Two-way ANOVA

809 (treatment $\times$ delay) revealed significant main effects of treatment after introduction

810 of hM4Di (treatment; $F_{(1,24)}=188, p=7.3 \times 10^{-13}$; delay; $F_{(2,24)}=57.3, p=7.3 \times 10^{-}$

$811^{10}$; interaction; $\left.F_{(2,24)}=10.9, p=4.3 \times 10^{-4}\right)$, but not before its introduction

812 (treatment; $F_{(1,24)}=0.03, p=0.86$; delay; $F_{(2,24)}=19.9, p=8.0 \times 10^{-7}$; interaction;

$\left.813 \mathrm{~F}_{(2,24)}=1.4, \mathrm{p}=0.26\right)$. C-E, Effects of oral administration of $300 \mu \mathrm{g} / \mathrm{kg}$ (orange) and

$8141,000 \mu \mathrm{g} / \mathrm{kg}$ (magenta), and intramuscular administration of $100 \mu \mathrm{g} / \mathrm{kg}$ (green) of

815 DCZ on task performance. The same data for the vehicle were plotted in each

816 panel (cyan). The behavioral tests were conducted $1-2 \mathrm{~h}$ following each

817 administration. In all conditions, two-way ANOVA (treatment $\times$ delay) revealed

818 significant main effects of treatment and delay, and interaction (100 $\mu \mathrm{g} / \mathrm{kg}$, i.m.;

819

treatment: $F_{(1,30)}=17.4, p=2.3 \times 10^{-4}$; delay: $F_{(2,30)}=41.6, p=2.2 \times 10^{-9}$;

820 interaction: $F_{(2,30)}=13.5, p=6.4 \times 10^{-5} ; 300 \mu \mathrm{g} / \mathrm{kg}$, p.o.; treatment; $F_{(1,30)}=60.9, p$

$821=1.1 \times 10^{-8} ;$ delay; $F_{(2,30)}=117.1, p=7.0 \times 10^{-14}$; interaction; $F_{(2,30)}=40.5, p=3.0$

$822 \times 10^{-9} ; 1000 \mu \mathrm{g} / \mathrm{kg}$, p.o.; treatment; $F_{(1,30)}=22.9, p=4.2 \times 10^{-5} ;$ delay; $F_{(2,30)}=62.7$,

$823 p=1.9 \times 10^{-11}$; interaction; $\left.F_{(2,30)}=21.4, p=1.7 \times 10^{-6}\right)$, indicating the delay-

824 dependent behavioral deficits. The data from two monkeys were pooled ( $N=6$

825 sessions; 3 sessions for each monkey). F-H, Time-course of behavioral effects of 
826 each administration. Only the correct rates of 30-s trials were plotted, as they were 827 most prominent, as shown in (C-E). Asterisks indicate significant differences 828 compared to vehicle administrations ( $p<0.05$, Welch's t-test, uncorrected). Error 829 bars represent s.e.m.

830 
831 Figure 5. Effects of $\mathbf{1 0 0 0} \mu \mathrm{g} / \mathrm{kg} \mathrm{DCZ}$ oral administration on behavioral tasks in 832 non-DREADD animals. A, Performance of delayed response task following oral 833 administration of vehicle (cyan) and $1000 \mu \mathrm{g} / \mathrm{kg} \mathrm{DCZ}$ (red) in a non-DREADD 834 animal (\#226). There was no significant difference between treatments (two-way 835 ANOVA, treatment $\times$ delay, treatment: $F(1,24)=0, p=1)$. B, Illustration of a 836 reward-size task. Each trial in this task began when the monkey touched a lever, 837 which was followed by the appearance of a visual cue signaling the size of the 838 upcoming reward (1, 2, 4 or 8 drops). To obtain the reward, the monkeys had to 839 release the lever when a visual target changed color from red to green. C, Example 840 behavioral data of reward size task for DCZ (1000 $\mu \mathrm{g} / \mathrm{kg}$, p.o.; red) and vehicle 841 administration session (cyan) obtained from a non-DREADD monkey (\#239). Two842 way ANOVA (treatment $\times$ reward size) revealed no significant difference $(p>0.05)$. 843 Error bars represent s.e.m. 
845 Figure 6. Chronic behavioral effect of daily DCZ administration across weeks.

846 A, Schedule of experiments. In a series of sessions, after 7 days of vehicle

847 administrations (Veh), DCZ was orally administered for 16 days, followed by 3 days

848 of vehicle, once each day. The monkey (\#245) was tested by delayed response

849 task over 5, 12 and 3 days, respectively. The series of testing were repeated twice.

850 Cyan and purple circles represent the drugs administered (vehicle or DCZ), and the

851 character "T" below the circles represents the days on which the behavioral tests

852 were conducted. B, Schedule of an experiment in one day. C, Correct rates plotted

853 as a function of days for vehicle (cyan) and DCZ (red) administrations for the 1st

854 (circle) and 2nd (triangle) series, respectively. There was significant difference

855 between vehicle and DCZ administrations (two-way repeated measures ANOVA

856 with treatment and schedule (1st and 2nd schedules), main effect of treatment,

$\left.857 \quad F_{(1,18)}=194, p=4.4 \times 10^{-11}\right)$. There was no significant difference between

858 performance during the former and latter periods (two-way repeated measures

859 ANOVA with timing (former vs latter) and schedule, main effect of timing, $F_{(1,10)}=$

$8600.03, p=0.87$ ), indicating that the impairment was consistent during the period of

861 administration. D, Plasma DCZ concentration plotted as a function of days for

862 vehicle (cyan) and DCZ (red) administrations for the same monkey (\#245). Data

863 were collected as with the behavioral data in (D), that is, $1 \mathrm{~h}$ after oral

864 administration of $300 \mu \mathrm{g} / \mathrm{kg} \mathrm{DCZ}$. Note that the DCZ concentration appeared to be

865 relatively higher than those of other monkeys in Fig. 1A. This might be attributable

866 to the difference of sampling conditions, i.e., under anesthesia or awake conditions 
867 (see Methods for details). It is also possible that oral administration of DCZ may be 868 less efficient for crossing the blood-brain barrier in animal \#245, since the PET data 869 suggests that brain uptake of DCZ in \#245 was relatively low compared to the other 870 animals (although it was still within a standard range; Fig. 3I). 


\begin{tabular}{|l|l|l|l|l|l|l|l|l|}
\hline ID & Species & Sex & Age (y) & Vector & Occupancy & PK & DCZ uptake & Behavior \\
\hline 201 & R & M & 10 & $\begin{array}{l}\text { hM4Di } \\
\text { OFC }\end{array}$ & & & $\checkmark$ & \\
\hline 221 & J & M & 7 & $\begin{array}{l}\text { hM4Di } \\
\text { OFC }\end{array}$ & & & $\checkmark$ & \\
\hline 226 & J & F & 8 & N/A & & $\checkmark$ & & $\checkmark$ \\
\hline 228 & R & M & 7 & N/A & & & $\checkmark$ & \\
\hline 229 & R & M & 5 & $\begin{array}{l}\text { hM4Di } \\
\text { dIPFC }\end{array}$ & & & $\checkmark$ & $\checkmark$ \\
\hline 237 & J & M & 7 & $\begin{array}{l}\text { hM4Di } \\
\text { amygdala }\end{array}$ & $\checkmark$ & & & \\
\hline 239 & J & M & 6 & N/A & & & & $\checkmark$ \\
\hline 240 & J & M & 6 & $\begin{array}{l}\text { hM4Di } \\
\text { OFC }\end{array}$ & & & $\checkmark$ & \\
\hline 245 & J & F & 7 & $\begin{array}{l}\text { hM4Di } \\
\text { dIPFC }\end{array}$ & & $\checkmark$ & $\checkmark$ & $\checkmark$ \\
\hline 248 & J & M & 4 & N/A & & $\checkmark$ & & \\
\hline 249 & J & M & 4 & N/A & & $\checkmark$ & & \\
\hline 253 & J & M & 5 & N/A & & & & $\checkmark$ \\
\hline Total & $12(\mathrm{R}, 2 / J, 10)$ & F,2; M,10 & & 6 & 1 & 4 & 6 & 5 \\
\hline
\end{tabular}

873

874 A tick indicates the subject was used in the experiment. PK, pharmacokinetics

875 study; OFC, orbitofrontal cortex; Species (J, Japanese; R, Rhesus), Sex (F,

876 Female; M, Male), Age (years). Note that monkeys \#229 and \#245 were used in

877 our previous studies (Nagai et al., 2020; Oyama et al., 2021).
878

879 

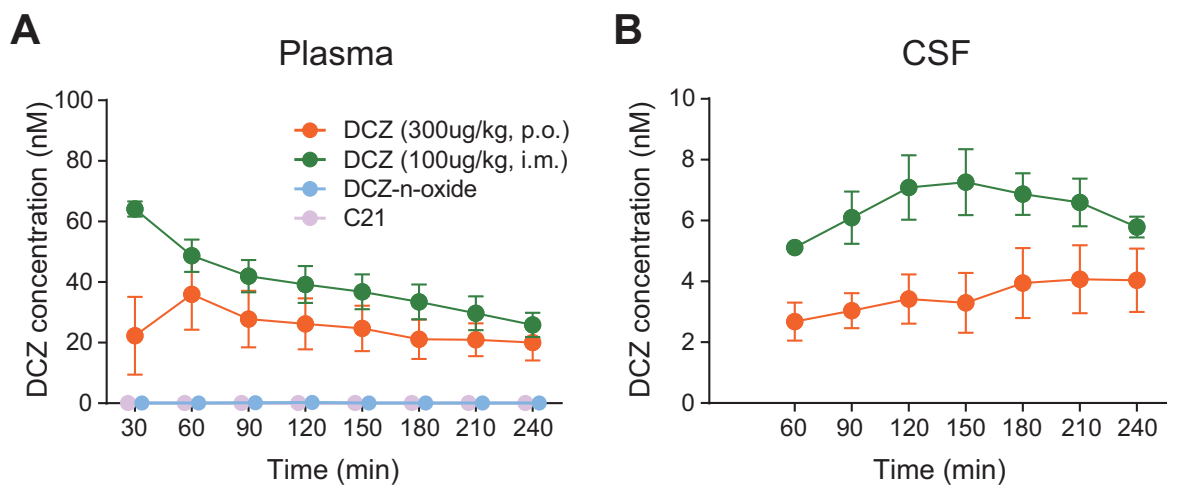
A

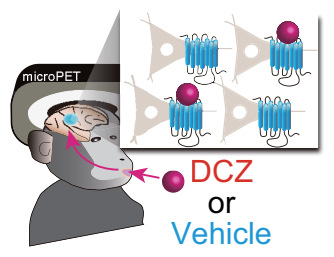

Pretreatment

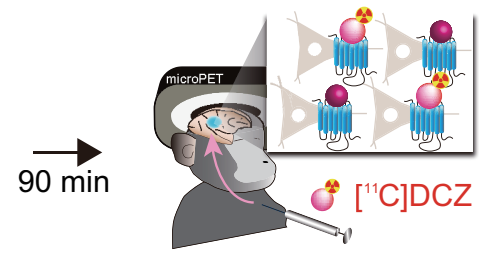

PET scan
B

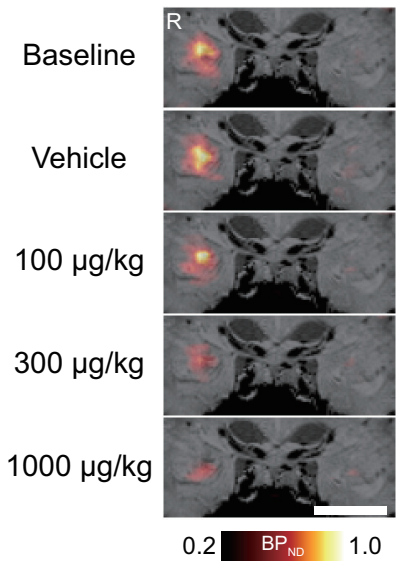

C

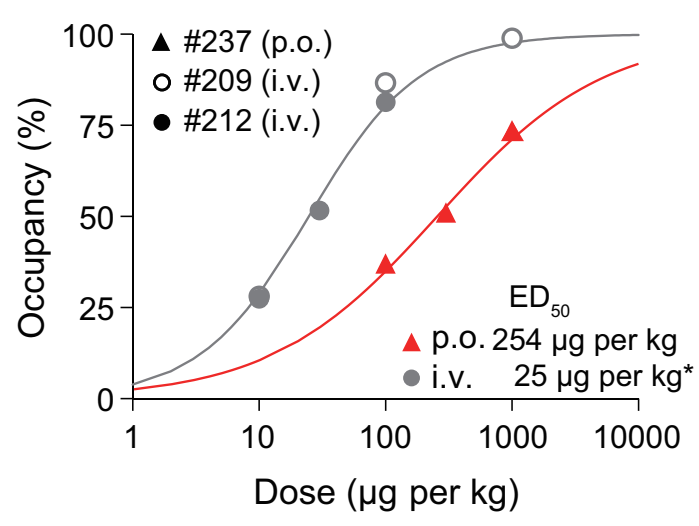



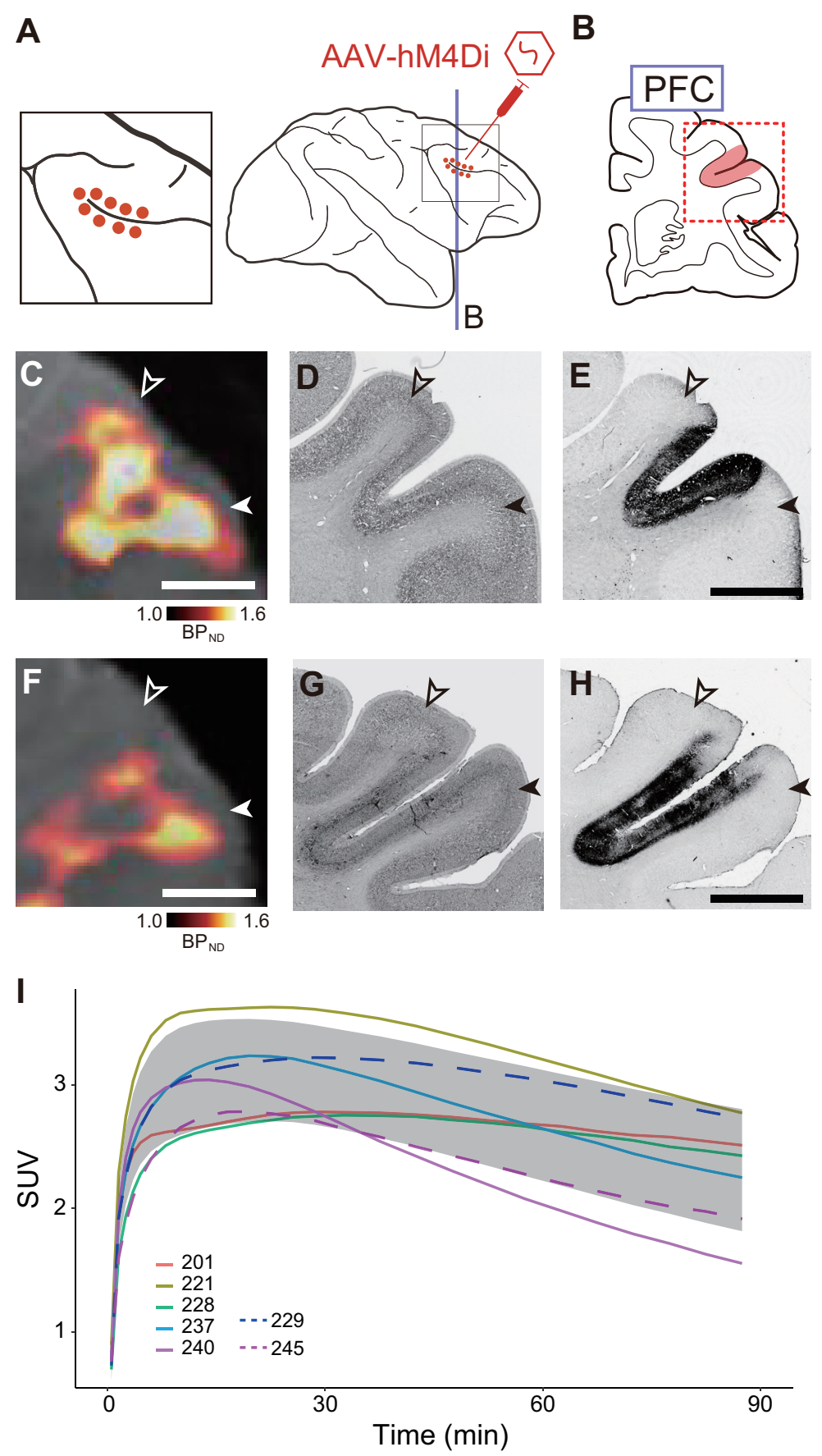
A

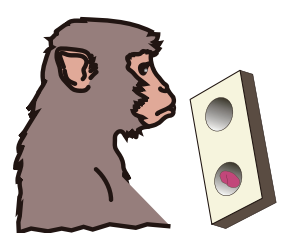

Cue

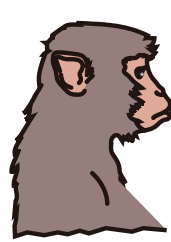

Delay

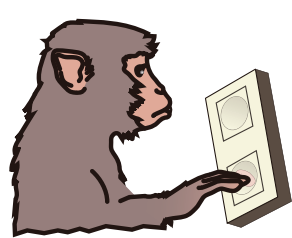

Response
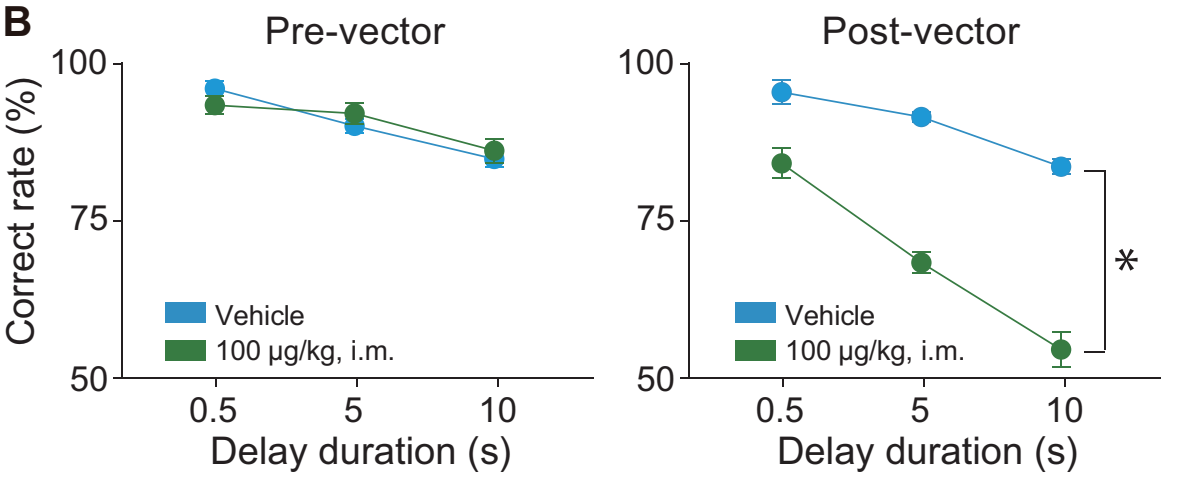

0

(1)

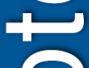

ㅇ.

(1)

0

0

$<$

0

(

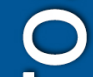

윽

(1)
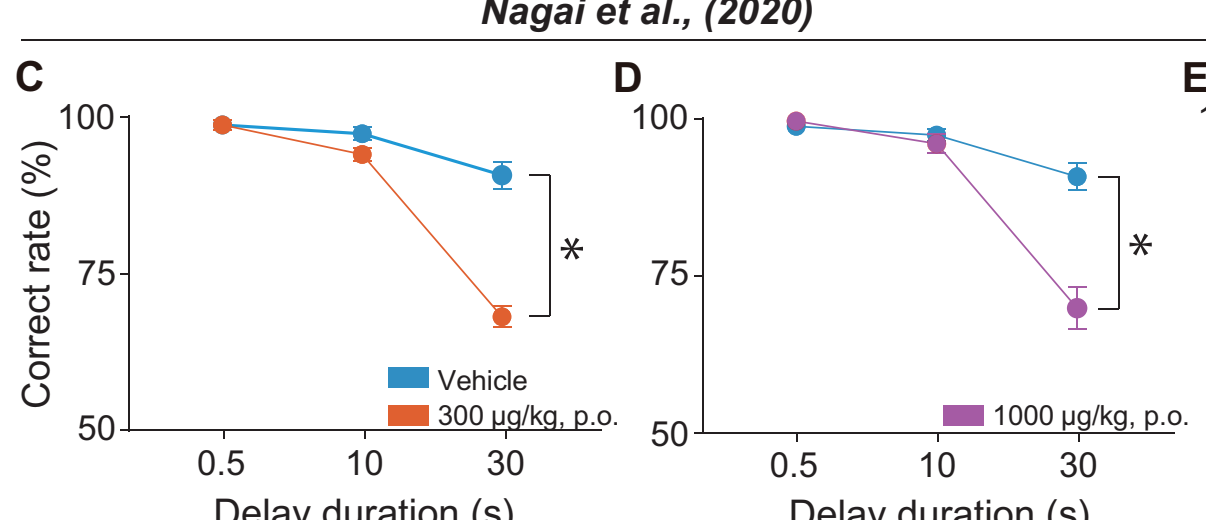

E

Delay duration (s)

Delay duration (s)
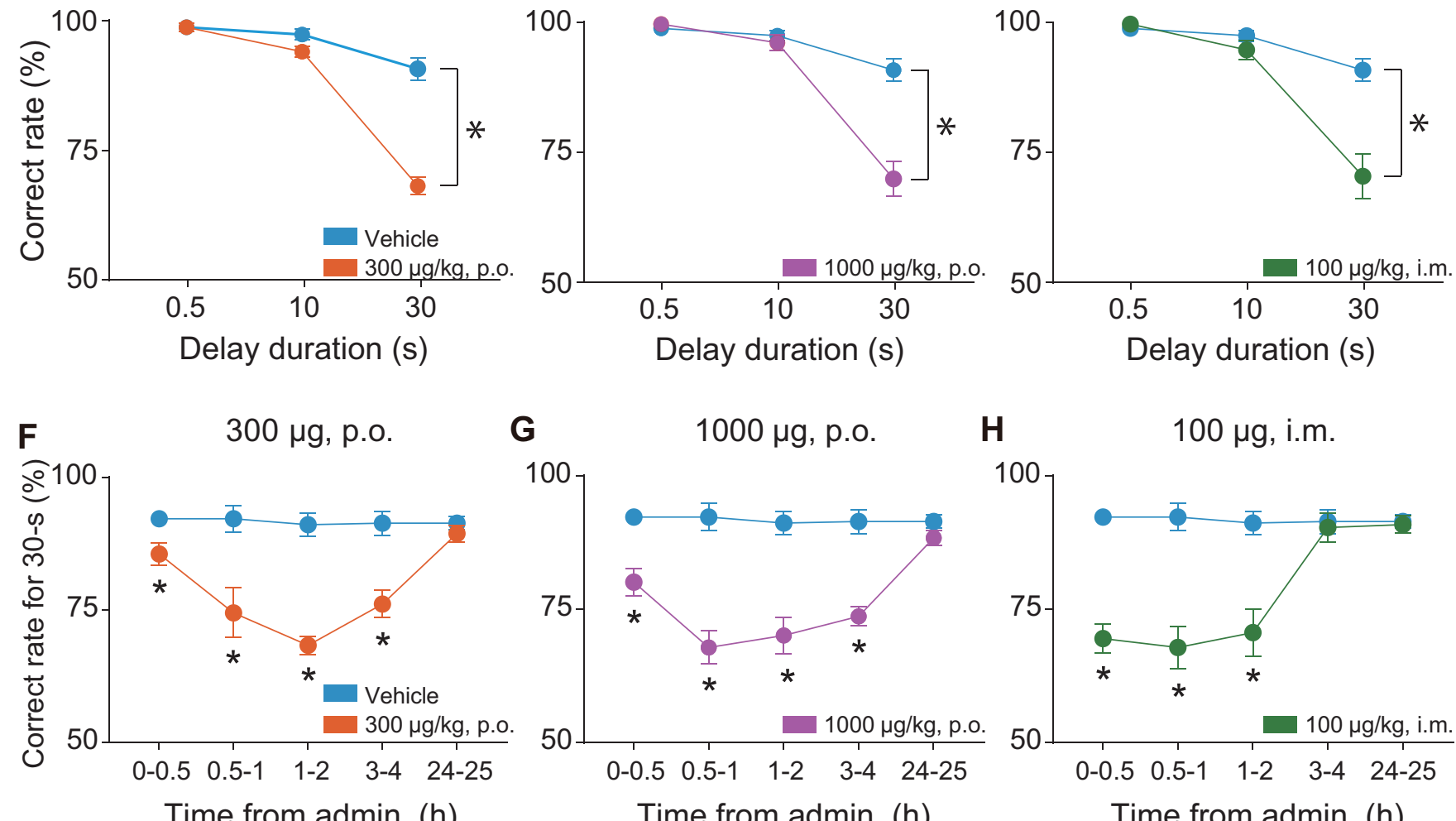

G

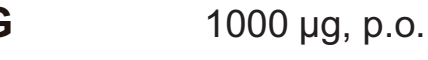

H
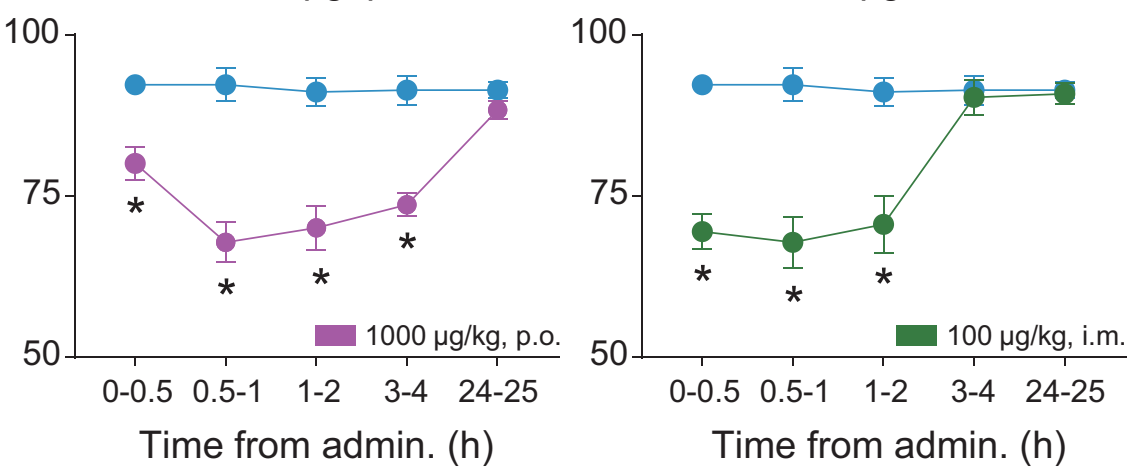


\section{A}

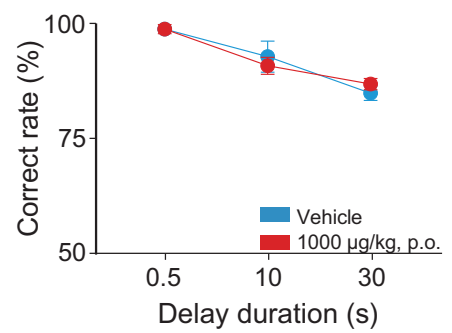

B

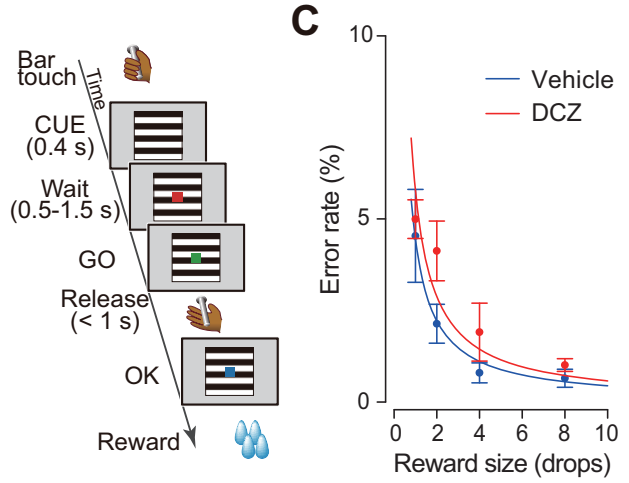




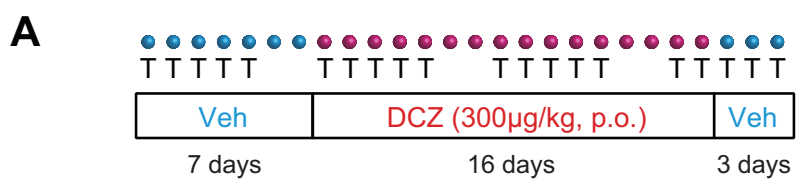

B

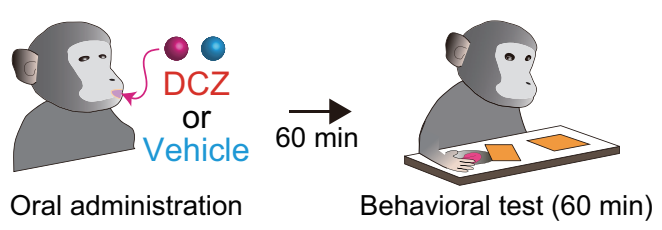

C

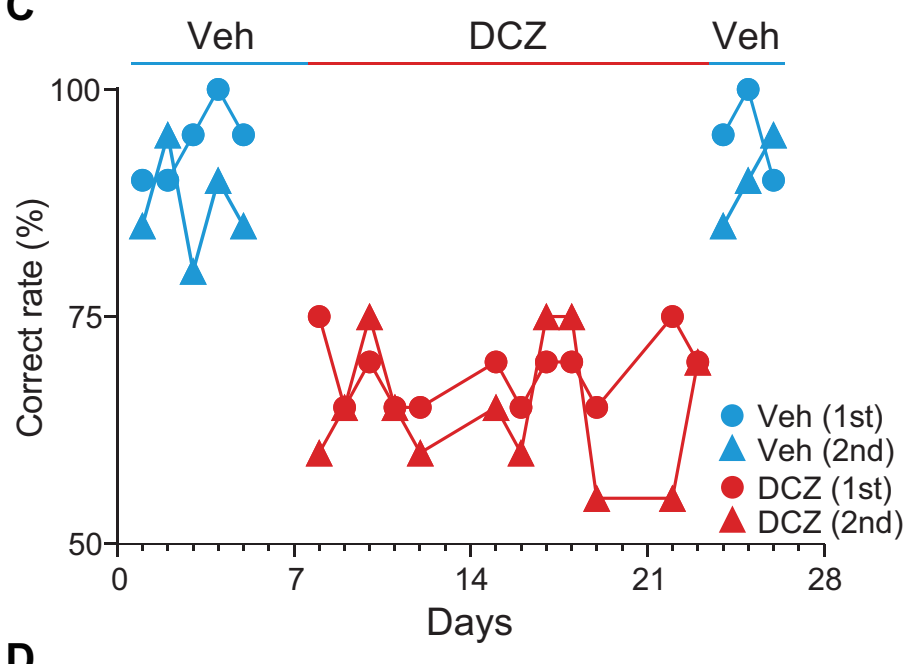

D

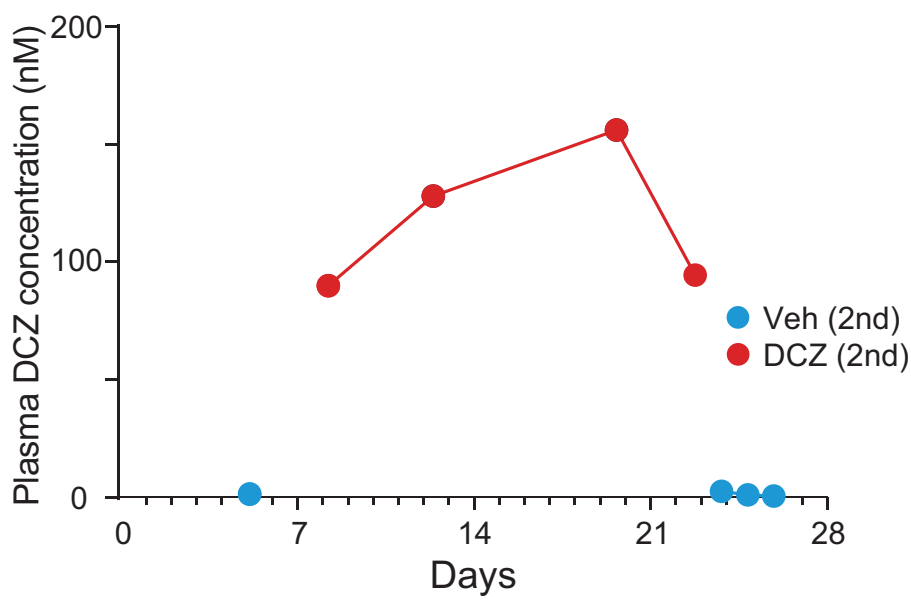

